\title{
An Improved Model of Snakes for Model-Based Segmentation *
}

\author{
Petia Radeva and Enric Martí \\ Computer Vision Center, \\ Universitat Autonoma de Barcelona, 08193 Bellaterra (Barcelona), Spain \\ e-mail: petia@upisun1.uab.es, enric@upisun1.uab.es
}

\begin{abstract}
The main advantage of segmentation by snakes consists in its ability to incorporate smoothness constraints on the detected shapes that can occur. Likewise, we propose to model snakes with other properties that reflect the information provided about the object of interest in a different extent. We consider different kinds of snakes, those searching for contours with a certain direction, those preserving an object's model, those seeking for symmetry, those expanding open, etc. The availability of such a collection of snakes allows not only the more complete use of the knowledge about the segmented object, but also to solve some problems of the existing snakes. Our experiments on segmentation of facial features justify the usefulness of snakes with different properties.
\end{abstract}

Keywords: snakes, elastic matching, model-based segmentation

\section{Introduction}

The successful automatic extraction of non-rigid objects in images is strongly depending on the existence of an object model that should be descriptive enough to embody common shape, but yet flexible to allow some degree of variation [3]. With this fact we can explain the common use of deformable templates used in model-based segmentation of flexible objects e.g. facial features [9]. Deformable templates are collections of parameterized curves (parabolae and circles) which dynamically adjust themselves to the image data in an energy-minimizing framework. Another segmentation approach based on energy minimization is the snake technique proposed by Kass et al. [5]. A snake is an elastic curve placed on an image, that deforms towards image features satisfying certain smoothness constraints.

The deformable templates approach has been mainly preferred because the classical snakes do not take into account any specific a priori knowledge available, have more parameters to update, and, hence, they are computationally slower [9]. In this paper we consider snakes with different properties, where these problems are considered. Moreover, in our collection of snakes the user can choose between

* In Proceedings of International Conference on Computer Analysis and Image Processing (CAIP'95), Prague, Czech Republic, September, 1995. 
snakes that reflect the knowledge about the object of interest at different level of concretion: from snakes guided by a model, throughout snakes using some general information (symmetry or contour orientation) about the object, till the classical snakes with little information about the object of interest.

The existence of such a collection not only allows to use the different kinds of information about the object of interest more completely, but also helps avoid some problems of classical snakes: attraction by spurious edges, degeneration, segmentation of concave shapes, etc. Finally, it is important to notice that for the snakes of our collection the parameters to be adjusted are less than these of the deformable models. Thus their computational cost is reduced.

Variations of the classical scheme of snakes has been suggested in various works $[1,4,7,8]$ in response to the need to solve some of the problems of the original snakes. Although some general similarity between the ideas for snakes with certain properties can be noted (e.g. removing spurious edges by discriminating by their orientation in [4]) in these cases the principal difference of the snakes proposed here consists in their simple implementation, carried out in the framework of the active contour models and providing better behaviour of the deforming snake. Thus, for example in case of considering the contour orientation, our snake is not attracted by edges with spurious direction instead of removing them after the deformation of the snake as it has been done in [4].

Our main goal is not to propose a modification of the classical snake to solve a given problem. In this paper we argue that disposing of a collection of snakes with different properties allows for many more possibilities in the process of segmentation. It is a concern of the expert to decide which snake or combination of snakes should be used given information about the object of interest, its scene and the original image.

The article is organized as follows: in section 2, we expose the fundamentals of the snakes. In section 3 , snakes with different properties are given. In section 4 we discuss the application of the different snakes for facial feature segmentation. Finally, conclusions are reported.

\section{Snake Model: Fundamentals}

A snake is a continuous curve that, from an initial state, tries to position itself dynamically on image features (e.g. edge points). It is deformed as a result of influence of external forces derived from edge points, while this deformation remains smooth due to the effect of internal forces. The internal forces minimize the internal energy of the snake given by the sum of the membrane energy (expressing the snake stretching) and of the thin-plate energy (expressing the snake bending): $E_{\text {int }}(u(s))=\alpha(s)\left|u_{s}(s)\right|^{2}+\beta(s)\left|u_{s s}(s)\right|^{2}$, where $u(s)=(x(s), y(s))$ is the snake curve [5]. The parameters of elasticity $\alpha$ and $\beta$ control the smoothness of the snake.

External forces are associated to a potential $P(x, y)$, which is defined in terms of the image gradient [5] or as a distance map of the edge points [2]. The snake moves due to the external forces trying to fall in a potential valley. 
The total snake energy is given by the functional of the energies sum:

$$
E_{\text {snake }}=\int_{0}^{1} E_{\text {int }}+E_{\text {ext }} d s=\int_{0}^{1} \alpha(s)\left|u_{s}(s)\right|^{2}+\beta(s)\left|u_{s s}(s)\right|^{2}+P(u(s)) d s
$$

\section{Library of Snakes with Different Properties}

Similarly to the snakes searching for smooth shapes, we modelize snakes with other properties that additionally improve the result in the segmentation process.

\subsection{Snake Selective with respect to Characteristic Orientation}

Though there exist various techniques to generate potentials, in all of them we have information in a point only about the distance of the closest image characteristic (usually, edge points). We would save many incorrect displacements if in a certain distance we have more information (e.g. its orientation) about the closest edge in order to decide whether or not to deform the snake towards it. The potential field of this kind of snake explicitly incorporates information about the gradient direction of the edge points [6]. It is defined by distance propagation using the following formula: $P(x, y)=c \log _{a}(1+d(x, y))$ where $d(x, y)$ is the distance of pixel $(x, y)$ until its closest edge point, and $a$ is a constant depending on the application. $c$ is a constant that propagates information about the orientation of the edges. It is 1 when the distance propagation is in the opposite direction to the gradient of the closest edge point, and -1 , otherwise.

Given the snake as a closed curve we define the external force as follows [6]:

$$
F(x, y)=\operatorname{sgn}(P(x, y))\left(\cos \phi P_{x}(x, y), \sin \phi P_{y}(x, y)\right)
$$

where $\phi$ is the angle of the normal directed towards the snake interior. Thus it can be shown that only edge points with suitable directions will attract the snake. From (1) it also follows that when the closest edge point for a given snake pixel has not the suitable direction, the external force repeals the snake from the closest contour. In some cases, this repulsion makes sense e.g. in the case of close objects if the snake has approached the neighbour object it should be moved in the opposite direction to find the contour of the object of interest. In other cases, e.g. in a scene of possible overlapping of objects, this fact is not valid. Then we define the external force as follows:

$$
\begin{aligned}
& F_{1}(x, y)= \begin{cases}\operatorname{sgn}(P(x, y)) \cos \phi P_{x}(x, y), & \text { if }-P_{x}(x, y) \cos \phi>0 \\
0, & \text { elsewhere. }\end{cases} \\
& F_{2}(x, y)= \begin{cases}\operatorname{sgn}(P(x, y)) \sin \phi P_{y}(x, y), & \text { if }-P_{y}(x, y) \sin \phi>0 \\
0, & \text { elsewhere. }\end{cases}
\end{aligned}
$$

In case of a partly non-attracted snake, this part of the snake is affected only by the internal force. If another part of the snake is moved to the correct contour, since the internal force preserves the entirety of the model, the former part can be displaced falling in a zone of a correct contour (Fig. 1). 


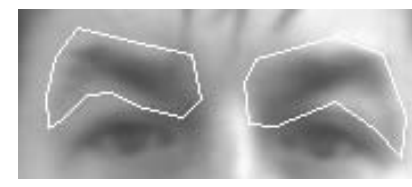

a

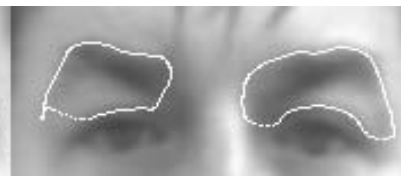

$\mathrm{b}$

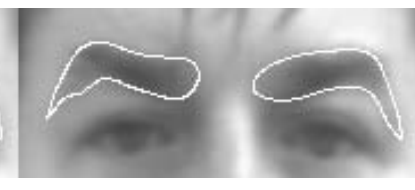

Fig. 1. Segmentation of eyebrows: initial snakes (a), result of the classical snake deformation (b), improved result of segmentation by snakes selective with respect to the edges' orientation (c).

\subsection{Snake Preserving the Initial Model}

In model-based segmentation a guidance by an object's model helps to the snake find only shapes that are close to the model. Therefore, the internal energy is determined as follows:

$$
E_{\text {int }}=\alpha\left(u_{s}(s)-u_{s}^{0}(s)\right)^{2}+\beta\left(u_{s s}(s)-u_{s s}^{0}(s)\right)^{2}
$$

where $u_{s}^{0}(s)$ and $u_{s s}^{0}(s)$ are the derivatives regarding the model contour. Thus, a structural information about the desirable shape of the snake is explicitly incorporated. The internal force of this kind of snakes attempts to compensate the changes caused by the external force and to preserve the object model controlled by the parameters of elasticity $\alpha$ and rigidity $\beta$. Moreover, it is easy to show [6] that the numerical solution of this kind of snake has the same amount of computations and properties as those of the classical snake.

One important consequence of the internal force definition (2) is that, trying to preserve the model shape, the snake never shrinks nor accumulates its parts. Using the internal energy (2) can also be considered as an alternative solution to the problem of spatial determination of the elasticity parameters. This local dependence of the parameters is necessary when some discontinuities should be allowed in certain parts of the snake. In case of model-based segmentation the proximal neigbourhood and the expected kind of discontinuity are available by the object model $u_{0}$ explicitly incorporated by the internal force.

\subsection{Symmetry-seeking Snake}

Designing symmetry-seeking snakes helps significantly ignore spurious characteristics in the image. Thus, to increase the global control on the snake deformation, we add a new term to the internal force of the snake definition. In case of symmetrical objects with respect to a given axis (e.g. the eyes), the internal force is defined as follows:

$$
E_{\text {int }}=\alpha u_{s}^{2}(s)+\beta u_{s s}^{2}(s)+c\left(u(s)-u^{s y m}(s)\right)
$$

where $\left.u^{s y m}(s)\right)$ stands for the symmetrical vector of the snake $u(s)$ with respect to the axis. The coefficient $c$ controls the strength of the symmetry constraint. 
Similarly, we model two snakes to segment symmetrical objects with respect to given axis (e.g. the eyes). In this case, for each object its symmetrical image $u^{s y m}$ is calculated. The segmentation of both objects is done simultaneously. The important point here is that segmentation of approximately symmetrical shapes (e.g. the mouth) is possible. That is why the segmentation of facial features by snakes provides more natural results than by deformable templates.

\subsection{Expanding Snakes}

Due to the tendency of the open snakes to shrink themselves once they have fallen in a potential valley, it is useful to modelize snakes able to expand themselves as well. The expanding effect is achieved in two steps; firstly, we make the ends of the snake expand (by $\alpha<0$ ). Thus, the snake ends go away from their neighbours. To keep the external energy low, the snake ends slide throughout the valley. The intermediate pixels keep the parameter $\alpha$ positive in order to not leave the valley. The parameter $\beta$ is kept positive for all pixels of the snake, because when expanding the snake, we are interested in a non bended snake curve. Secondly, we fix the end points and make the snake deform till its convergence in order to avoid contraction and instable movement.

\subsection{Snakes with Evenly Distributed Pixels}

This kind of snake differs from the classical one in that in each step of its deformation it is taken care of by the pixels so that their distance keeps equal. One of the applications of this snake is in segmentation of concave objects. Often, detecting the concave location of the contour needs more stretching of the snake than the internal energy allows. Depending on the initial position sometimes the pixels of certain parts of the snake accumulate. This makes the other parts of the snake expand too much. However, if the snake is deforming keeping the distances between the pixels equal, this problem is avoided.

\section{Application}

We have applied our snakes for features segmentation in facial images. 26 images are considered where the eyebrows, pupils, eyelashes and the mouth are to be detected. The conditions on the facial images were to allow $20 \%$ deviation in scale, orientation and translation. The background is supposed to be approximately homogeneous. Depending on the feature, different snakes are used in a sequence of stages in the segmentation process.

In Fig. 2 the consecutive steps of mouth segmentation are given. Firstly, we search for the line between the lips by an open classical snake deformed on a potential generated from valley points. To detect all the line between the lips, an expanding snake is used. Afterwards, a symmetry-seeking snake is applied on the edge potential to find the symmetrical external borders of the lips. When using the darkness of the lips, only edge points with suitable directions are considered, 
applying the snake selective with respect to the edge orientations. Finally, to capture the concavity of the top lip a snake with evenly distributed pixels is applied. The results have natural shape due to the good adjust of the snake to the image data and complete use of the knowledge about the feature. Moreover, the computational cost is kept low and the rate of success of segmentation is high $(89 \%)$.

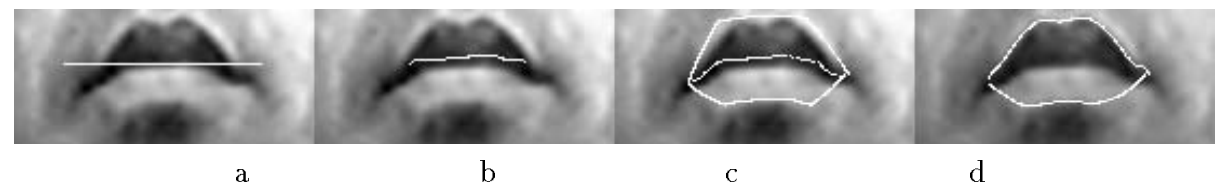

Fig. 2. Segmentation of a mouth: initial snake corresponding to the line between the lips (a), deformed classical snake (b), expanded snake and initial model of the external borders (c), final deformation by snakes selective respect the contours' orientation (d)

\section{Conclusions}

Despite the promising segmentation results achieved by the classical snake, the need of its improvement is suggested by the existence of various modifications. The proposal exposed above is that instead of replacing the classical snake with an improved version, the availability of a collection of snakes with different properties gives more possibilities to the expert to choose the snake or combination of snakes that best correspond to the application problem and the image data. Besides, we show that in this way many common problems of the snakes can be solved or compensated in the segmentation process. This article is an attempt to propose a restricted collection of snakes, to show how different types of information about the object can be implemented, to justify their use for model-based segmentation and to give a brief idea about how a combination of snakes can be applied in problems such as facial feature segmentation.

\section{References}

1. Ch.-W. Chen and Ch.-L. Huang, Int. J. of PRAI, Vol. 6, No. 4, pp. 571-594, 1992.

2. I. Cohen, L. D. Cohen and N. Ayache, CVGIP: IU, Vol.56, pp.242-263, 1992.

3. G. Chow and X. Li, Pattern Recognition, Vol. 26, No. 12, 1993, pp. 1739-1755.

4. P. Fua and Y. G. Leclerc, Machine Vision and Applications, Vol. 3, pp. 45-56, 1990.

5. M. Kass and A. Witkin and D. Terzopolous, Proc. of ICCV, 1987.

6. P. Radeva, Master Thesis, UPIIA, UAB, September, 1993.

7. D. Terzopoulos, J. Platt, A. Barr and K. Fleisher, Comp. Graphics, Vol. 21, 1987.

8. D. Terzopoulos, A. Witkin and M. Kass, Art. Intel., No 36, 1988, pp. 91-123.

9. A. L. Yuille, P. W. Hallinan and D. S. Cohen, IJCV, Vol. 8, no. 2, 1992. 
This article was processed using the $\mathrm{IAT}_{\mathrm{E}} \mathrm{X}$ macro package with LLNCS style 